\title{
Colored HOMFLY and generalized Mandelbrot set
}

\author{
Ya. Kononov ${ }^{d}$ and A. Morozov ${ }^{a, b, c}$ \\ a Theory Department, ITEP, \\ B. Cheremushkinskaya, 25, Moscow, 117218 Russia \\ ${ }^{b}$ Physics Departement, National Research Nuclear University MEPhI, \\ Kashirskoe sh., 31, Moscow, 115409 Russia \\ ${ }^{c}$ Math. Physics Lab, Institute for Information Transmission Problems, \\ B. Karetny 19, build. 1, Moscow, 127051 Russia \\ ${ }^{d}$ Math. Departement, HSE, \\ Vavilova, 7, Moscow, 117312 Russia \\ E-mail: yashakon@mail.ru, morozov@itep.ru
}

ABSTRACT: Mandelbrot set is a closure of the set of zeroes of $\operatorname{resultant}_{x}\left(F_{n}, F_{m}\right)$ for iterated maps $F_{n}(x)=f^{\circ n}(x)-x$ in the moduli space of maps $f(x)$. The wonderful fact is that for a given $n$ all zeroes are not chaotically scattered around the moduli space, but lie on smooth curves, with just a few cusps, located at zeroes of $\operatorname{discriminant}_{x}\left(F_{n}\right)$. We call this phenomenon the Mandelbrot property. If approached by the cabling method, symmetrically-colored HOMFLY polynomials $H_{n}^{\mathcal{K}}(A \mid q)$ can be considered as linear forms on the $n$-th "power" of the knot $\mathcal{K}$, and one can wonder if zeroes of resultant $q^{2}\left(H_{n}, H_{m}\right)$ can also possess the Mandelbrot property. We present and discuss such resultant-zeroes patterns in the complex- $A$ plane. Though $A$ is hardly an adequate parameter to describe the moduli space of knots, the Mandelbrot-like structure is clearly seen - in full accord with the vision of hep-th/0501235, that concrete slicing of the Universal Mandelbrot set is not essential for revealing its structure.

KEywords: Topological Strings, Chern-Simons Theories, Stochastic Processes

ARXIV EPRINT: 1510.01252 


\section{Contents}

1 Introduction 1

2 Technicalities 4

3 Pictorial evidence 5

4 Meditation: how special are sequences with Mandelbrot property? $\quad 7$

5 Fundamental HOMFLY and HOMFLY for other transposition-symmetric diagrams

6 More on the role of the special polynomials 10

7 Hopes for divisibility: differential expansion 12

8 Julia bundle $\quad 14$

9 Conclusion 18

\section{Introduction}

The purpose of this paper is to establish, at least phenomenologically, a clear relation between the two celebrated entities from the "opposite" corners of theoretical physics: Mandelbrot sets [1, 2], belonging to the world of discrete-time dynamical systems, and colored HOMFLY polynomials [3-10], providing the most important kind of knot invariants. Formulation and analysis of this relation is made possible by the algebro-geometric reformulation of Mandelbrot theory in [11, 12] and by the recent progress in understanding of symmetric HOMFLY, originated in $[13,14]$.

Mandelbrot set is a famous example of fractal structure, provided by consideration of iterated maps $f^{\circ n}(x)=f\left(f^{\circ(n-1)}(x)\right)$, which from the point of view of dynamical systems describe evolution in the discrete time $n$. If the shape of the map $f(x)$ is characterized by additional parameters (moduli) $c$, then the distribution of stable orbits in the $c$-space is described by the famous pattern (captured from [15] for the basic example $f_{c}(x)=x^{2}+c$ ):

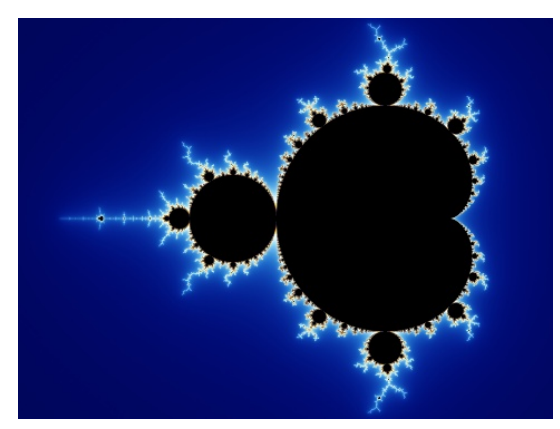


From a programmer's perspective, shown in black are the values of complex $c$, for which the sequence $f_{c}^{o n}(x=0)$ does not tend to $x=\infty$ (the choice of initial point $x=0$ is not always adequate - see [16] for improved algorithm). This, however, explains nothing about peculiar - and, in fact, universal - structure in the picture. What explains, is its algebro-geometric description in $[11,12]$ (see also [16, 17] and [18]).

Namely, consider a shifted iteration $F_{n}(x)=f^{\circ n}(x)-x$, its zeroes in $x$-space describe the closed orbits of length $n$. These orbits are stable, provided $\left|\frac{d f^{\circ n}}{d x}\right|<1-$ and this is true or not, depending on the value of $c$. The two equations

$$
\left\{\begin{array}{l}
F_{n}(x)=0 \\
\left|\frac{d f^{\circ n}}{d x}\right|=1
\end{array}\right.
$$

describe an almost-everywhere-smooth curve $L_{n}$ in the complex-c plane (perhaps, made of several components). In particular, the central cardioid in the picture is

$$
c=\frac{1}{2} e^{i \theta}-\frac{1}{4} e^{2 i \theta} \Longleftarrow\left\{\begin{array}{rl}
x^{2}+c-x & =0 \\
2|x| & =1
\end{array} .\right.
$$

The Mandelbrot set (or, if one prefers, its boundary) is actually a collection of these curves.

This is, however, not yet the needed algebro-geometric description. To get it, we further note that stability of the $n$-th orbit is lost when a Feigenbaum cycle-multiplication [19-21] occurs, and what gets stable is the orbit of the order $(k n)$ with some integer $k$. Such bifurcation happens at zeroes of $\operatorname{resultant}_{x}\left(F_{n}, F_{k n} / F_{n}\right)$, which densely populate the curve $L_{n}$ - and therefore $L_{n}$ can be considered as a closure of the set of these zeroes for all $k$. Cusp singularities of $L_{n}$ are actually located at zeroes of $\operatorname{discriminant}_{x}\left(F_{n}\right)$. In this description the central cardioid appears as

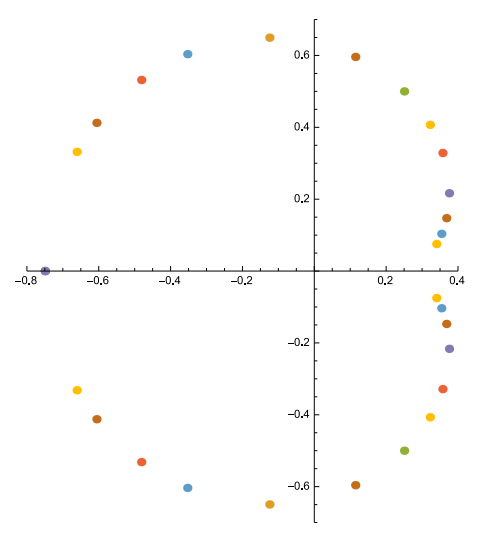

This description motivates the following definition:

A sequence of polynomials $P_{n}(x \mid c)$ has a Mandelbrot property, if the zeroes of resultants $R_{n, m}(c)=\operatorname{resultants}_{x}\left(P_{n}, P_{m}\right)$ in the space $\mathcal{M}$ of moduli $c$ for all $m$ belong to a variety $L_{n}$ of non-vanishing real codimension in $\mathcal{M}$. 
In fact, Mandelbrot property is very sensitive to details. If, for example, we choose a wrong definition of $F_{n}=f^{\circ n}(x)-\alpha x$ with $\alpha \neq 1$, no smooth curves emerge: for, say, $\alpha=0$ (green) and $\alpha=2$ (black) we get

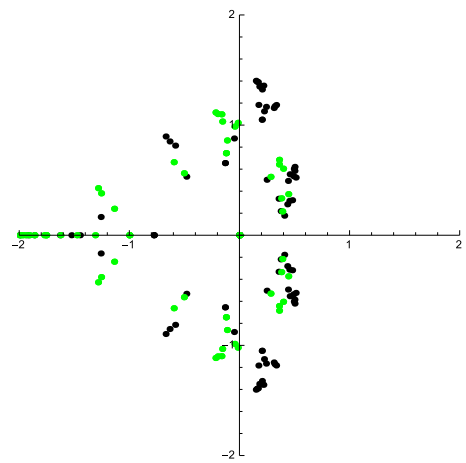

Thus Mandelbrot property is really distinguished: it is true for the right objects - but is easily violated for the wrong ones.

As argued in [11, 12], Mandelbrot property is universally true for sequences of shifted iterated maps $F_{n}$ - whatever is the variety of functions (maps) $f_{c}(x)$. The natural question is if there are other interesting examples.

What we demonstrate in the present paper, is that the sequence of symmetrically colored HOMFLY polynomials $H_{n}(q)$ has a good chance to possess Mandelbrot property for arbitrary knot, even if the role of moduli is played by the second HOMFLY variable $A$.

First of all, this is almost a fact, which we try to make pictorially-obvious in section 3 . We then discuss a more theoretical evidence in sections $5-8$.

Second, one can wonder, if this fact could be expected - or, in other words, if there is anything in common between iterated maps and colored HOMFLY, i.e. between iterations and colorings. In fact, there is.

If the map $x \longrightarrow f(x)$ was just linear, $x \longrightarrow f \cdot x$ (this is quite an interesting example, if $x$ is a vector, and $f$ - a matrix), then iteration is just a matrix product: $f^{\circ n} \sim f^{n}$, and the latter can be embedded into a tensor product $f^{\otimes n}$. Generalizing (or, perhaps, speculating) - even for non-linear maps one can consider iterations as some sophisticated projection functor from the tensor product:

$$
f^{\circ n}=\mathcal{P}_{\text {ite }}\left(f^{\otimes n}\right) .
$$

One can now speculate that Mandelbrot property is the one of the projector - and one can ask if there are other projectors, besides $\mathcal{P}_{\text {ite }}$ which possess this property.

In knot theory cabling method [22] allows one to consider colored HOMFLY as certain projection acting on the $n$-th "power" of the knot $\mathcal{K}$ :

$$
H_{n}^{\mathcal{K}}=\mathcal{P}_{\text {col }}\left(\mathcal{K}^{\sqcup n}\right) .
$$

Our claim in this paper is that $\mathcal{P}_{\text {col }}$ also has (a good chance to possess) the Mandelbrot property.

Third, why $A$ is considered as a modulus? Of course, it would be more natural to look at the moduli space of knots. What we do in this paper is just the search "under the 
lamp". Due to numerous recent advances [13, 14, 23-28], dependence of symmetricallycolored HOMFLY on $A$ is nowadays described in sufficiently clear and explicit way to make computer experiments and even some analytical studies. This is not yet so easy for $\mathcal{K}$-dependence - though a way is now open by the suggestions in $[29,30]$. However, if one accepts the concept of the Universal Mandelbrot set [11, 12], concrete choice of a single modulus (slicing) to look at is not too essential: Mandelbrot property will show up in each particular slicing - and from this point of view the perhaps-arbitrary choice of $A$ is not a problem.

Forth — what are the implications of our conjecture? We prefer to refrain from further speculations at this stage - despite there can be many. It is already sufficient that too well-developed branches of theory are now connected, and the exchange of ideas and concepts can now take place. Moreover, already the simplest examples we look at below, demonstrate that resultants know a lot about other non-trivial properties of knot invariants — like defects [31] of differential expansions [32-35] — what supports our belief that the relation to Mandelbrot world is a deep property and not just an amusing observation or a joke. This means that further work is needed to appreciate the real significance of this newly-emerging research direction, which finally extends the still-mysterious Mandelbrot property beyond a single example. Time is needed to put the right accents and provide relevant interpretations.

\section{Technicalities}

We remind that resultant is the function of coefficients of the system of homogeneous non-linear equations, which vanishes whenever the system has non-vanishing solution:

$$
\vec{F}(\vec{x})=0, \quad \vec{x} \neq 0 \quad \Leftrightarrow \quad \operatorname{resultant}_{\vec{x}}(\vec{F}) \neq 0 .
$$

Resultants - and discriminants, their close relatives, - are non-linear analogues of determinants. If known, they make the theory of non-linear equations as transparent as it is for linear ones - and the study of resultants and discriminants is the main topic in non-linear algebra [18, 36-42]. The present knowledge, however, remains restricted - and the problem attracts much less attention and effort than it deserves.

Fortunately, in the case of a single variable $x$ (i.e. two variables in homogeneous formulation), which we deal with in the present paper, the story is elementary - see any textbook on algebra for explicit formulas, e.g. section 3.3.3 of [18]. Discriminant of a polynomial $P(x)=\sum_{i=0}^{n} a_{i} x^{i}=\prod_{i}\left(x-x_{i}\right)$ is equal to

$$
\operatorname{discriminant}_{x}(P)=\prod_{i<j}\left(x_{i}-x_{j}\right)^{2}
$$

and it is a polynomial in the coefficients $a_{i}$. Similarly, a resultant if two polynomials $P_{1}(x)=\prod_{i}\left(x-x_{i}^{(1)}\right)$ and $P_{2}(x)=\prod_{j}\left(x-x_{j}^{(2)}\right)$,

$$
\operatorname{resultant}_{x}\left(P_{1}, P_{2}\right) \sim \prod_{i, j}\left(x_{i}^{(1)}-x_{j}^{(2)}\right)
$$


is a polynomial in $a_{i}^{(1)}$ and $a_{j}^{(2)}$, equal to determinant

$$
\operatorname{resultant}_{x}\left(P_{1}, P_{2}\right)=\operatorname{det}\left(\begin{array}{ccccccc}
a_{0}^{(1)} & a_{1}^{(1)} & a_{2}^{(1)} & a_{3}^{(1)} & \ldots & 0 & 0 \\
0 & a_{0}^{(1)} & a_{1}^{(1)} & a_{2}^{(1)} & \ldots & 0 & 0 \\
0 & 0 & a_{0}^{(1)} & a_{1}^{(1)} & \ldots & 0 & 0 \\
& & & \ldots & & \ldots & \\
0 & 0 & 0 & 0 & \ldots & a_{n_{1}}^{(1)} & 0 \\
0 & 0 & 0 & 0 & \ldots & a_{n_{1}-1}^{(1)} & a_{n_{1}}^{(1)} \\
a_{0}^{(2)} & a_{1}^{(2)} & a_{2}^{(2)} & a_{3}^{(2)} & \ldots & 0 & 0 \\
0 & a_{0}^{(2)} & a_{1}^{(2)} & a_{2}^{(2)} & \ldots & 0 & 0 \\
0 & 0 & a_{0}^{(2)} & a_{1}^{(2)} & \ldots & 0 & 0 \\
& & & \ldots & & \ldots & \\
0 & 0 & 0 & 0 & \ldots & a_{n_{2}}^{(2)} & 0 \\
0 & 0 & 0 & 0 & \ldots & a_{n_{2}-1}^{(2)} & a_{n_{2}}^{(2)}
\end{array}\right)
$$

and matrix has the size $n_{1}+n_{2}$.

The second ingredient of our consideration - colored HOMFLY polynomials - can be taken from the database in [43]: they are result of various calculations by a variety of modern methods, see [23-30] and references therein. HOMFLY in topological framing are actually Laurent polynomials, and to apply standard definitions of resultants, they should be multiplied by a certain power of $q$ to become ordinary polynomials — what is always assumed in this paper.

\section{Pictorial evidence}

With this data we can easily plot zeroes or colored HOMFLY resultants. Zeroes of resultant $_{q^{2}}\left(H_{1}^{4_{1}}, H_{k}^{4_{1}}\right)$ for the figure eight knot $\mathcal{K}=4_{1}$ :

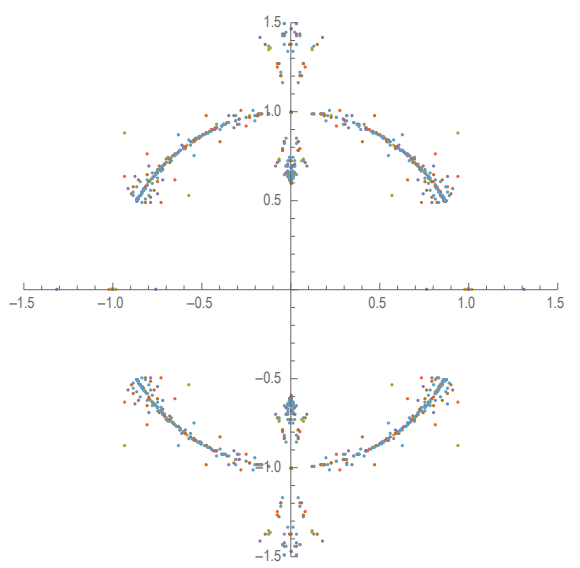


and those of resultant $q^{2}\left(H_{2}^{4_{1}}, H_{k}^{4_{1}}\right)$ :

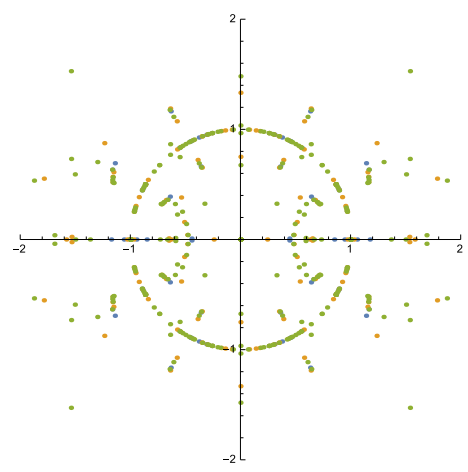

We see that instead of being chaotically scattered all over the complex $A$-plane, these zeroes form certain one-dimensional structures, i.e. exhibit the Mandelbrot property. Note, that the two segments of the unit circle in the first picture do not form a closed curve and can not serve as a boundary of any 2-dimensional domain - as it was in the Mandelbrot case. Still, the second picture extends these segments to a bigger fraction of the unit circle, and zeroes of all the resultants $R_{k, l}$ seem to populate densely the entire closed curve.

Situation for more complicated knots can look less convincing: the same plots for the defect-2 knot $\mathcal{K}=7_{1}$
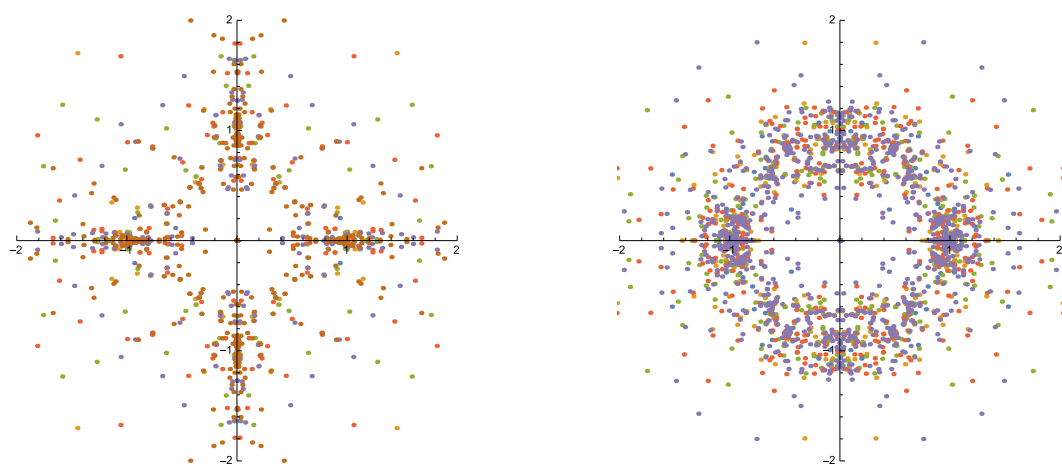

look more like full 2-dimensional patterns. However, this is only because of the poor resolution. Looking closer, we see, for example:

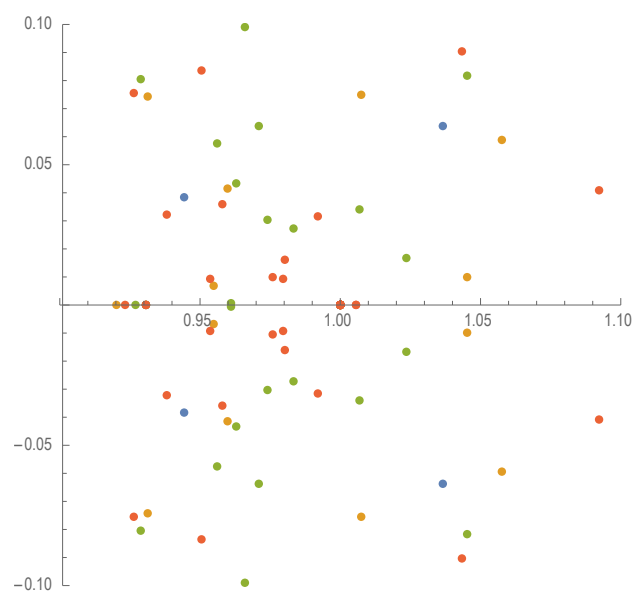

For more details and examples see [44]. 


\section{Meditation: how special are sequences with Mandelbrot property?}

While pictures in the previous section obviously reveal some correlation between zeroes of different resultants of colored HOMFLY polynomials, they are rather far from the clarity of (1.1) and (1.4) and can hardly be treated as an experimental proof the Mandelbrot property. At best they resemble (1.5) rather than (1.4), what can imply that HOMFLY polynomials deserve to be properly shifted to improve the situation. In the case of iterated maps there was a distinguished shift $-x$, dictated either by the formulation of the problem (search for closed orbits) or, by an algebraic criterium of divisibility of $F_{k n}$ by $F_{n}$, which a sort of "ties" at least some zeroes of $F_{k n}$ to those of $F_{n}$ and helps to choose the proper resultants. For HOMFLY the story is different: there is no clear way to make a shift, which would make $H_{k n}$ divisible by $H_{n}$. Still, some naive shifts improve the pattern, though not drastically enough. For example, a natural option is to shift $H_{n}$ by one, and here is what we get:

- roots of resultant $q^{2}\left(\frac{H_{2}^{3_{1}}-1}{\{A / q\}}, \frac{H_{j}^{3_{1}}-1}{\{A / q\}}\right)$ :

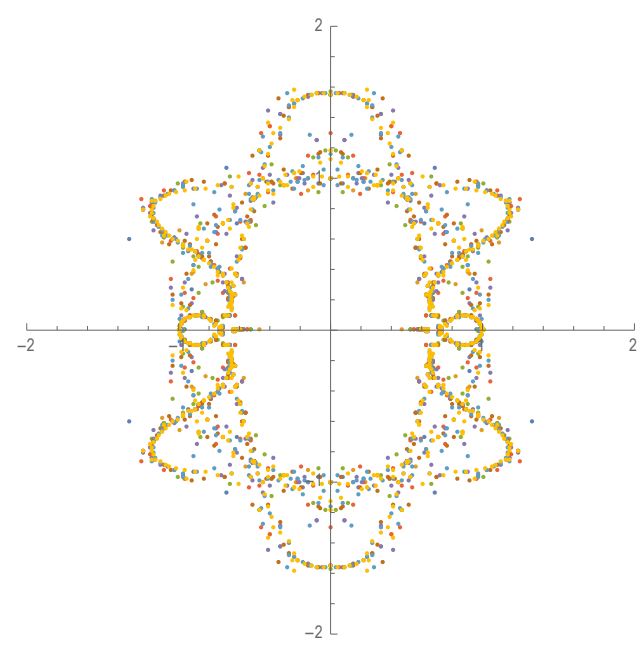

- roots of resultant $q^{2}\left(\frac{H_{2}^{3_{1}}-H_{1}^{3_{1}}}{\{A / q\}}, \frac{H_{j}^{3_{1}}-H_{1}^{3_{1}}}{\{A / q\}}\right)$ :

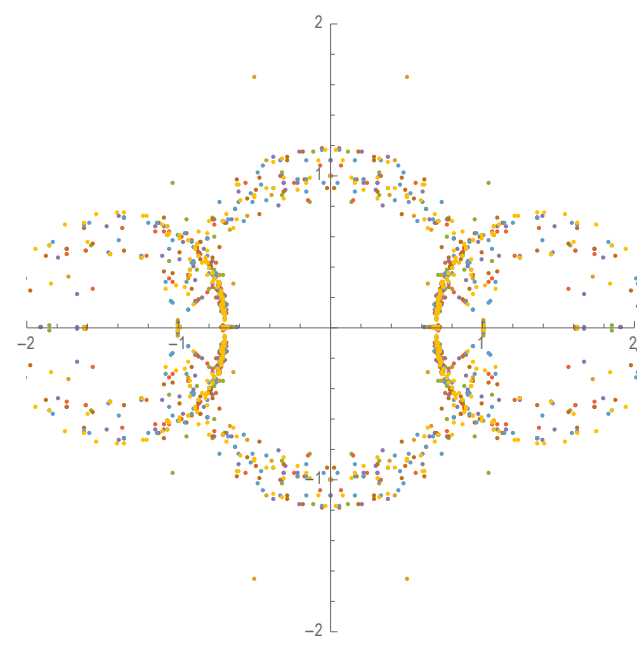


One can now ask a natural question - if these somewhat obscure pictures manifest anything at all: perhaps, patterns of this quality are provided by just arbitrary sequence of polynomials. To illustrate the actual state of affairs, we consider not an arbitrary, but a very special sequence $\left\{G_{n}(x, z)\right\}$, provided by the $t$-expansion of the generating function

$$
\exp \left(\sum_{k=1}^{m} t^{k} P_{k}(x, z)\right)=\sum_{n=0}^{\infty} t^{n} G_{n}(x, z)
$$

i.e. $G_{n}$ are symmetric Schur functions of the polynomial-valued time-variables $P_{k}-$ and plot the zeroes of $\operatorname{resultant}_{x}\left(G_{k}, G_{l}\right)$ in the complex- $z$ plane.

At $m=1$ all $G_{n} \sim P_{1}^{n}$ and all resultants are just vanishing.

At $m=2$ they all reduce to a single resultant ${ }_{x}\left(P_{1}, P_{2}\right)$ and there is nothing interesting.

However, starting from $m=3$, the situation changes: e.g. for

$$
\begin{aligned}
& P_{1}=x+z \\
& P_{2}=x^{2}+z^{2} \\
& P_{3}=x^{3} z^{2}
\end{aligned}
$$

the pattern of resultant zeroes looks as follows:

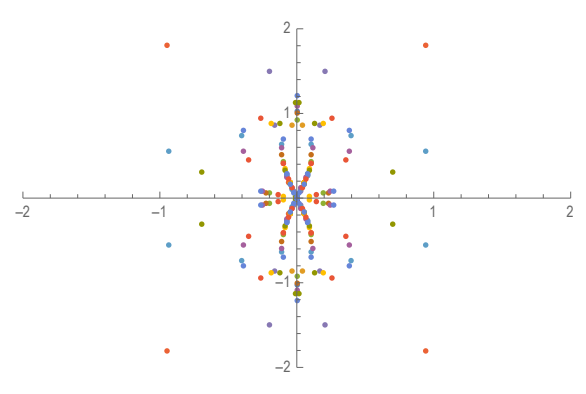

while for

$$
\begin{aligned}
& P_{1}=z^{3}+z^{2}-x^{2} z \\
& P_{2}=z^{5}-x^{7} z^{3} \\
& P_{3}=x^{3} z^{2}+x^{2} z^{4}
\end{aligned}
$$

it becomes

We clearly see that with increase of $m$ and/or of the complexity of the polynomials $P_{k}$ the one-dimensional structures quickly dissolve in two-dimensional clouds and Mandelbrot 
property, if at all present, gets destroyed - and this happens already for a very special sequence, provided by a Schur transform from just a few simple polynomials.

In fact, for $m=3$ a zero of $\operatorname{resultant}_{x}\left(G_{m}, G_{n}\right)$ is associated with a common solution of two equations

$$
\left\{\begin{array}{l}
P_{2}=a_{m n} \cdot P_{1}^{2} \\
P_{3}=b_{m n} \cdot P_{1}^{3}
\end{array}\right.
$$

i.e.

$$
\operatorname{resultant}_{x}\left(G_{m}, G_{n}\right) \sim \operatorname{resultant}_{x}\left(P_{2}-a_{m n} \cdot P_{1}^{2}, P_{3}-b_{m n} \cdot P_{1}^{3}\right)
$$

where $a$ and $b$ are numerical constants, depending on $m$ and $n$ - and it is the diversity of the coefficients $a_{m n}$ and $b_{m n}$ which is responsible for the lack of Mandelbrot property. A detailed study of this simple example is of its own interest, but it would take us too far away from the main subject of the present paper.

Instead we now return to HOMFLY resultants and proceed from pictures of section 3 to formulas. We do not do much in the present paper, but the kind of questions to address should be clear from the simple examples below.

\section{Fundamental HOMFLY and HOMFLY for other transposition-sym- metric diagrams}

Colored HOMFLY polynomials are defined for arbitrary representation (Young diagram) $R$ and they possess the important symmetry: transposition of $R$ is equivalent to inversion of $q^{2}$ :

$$
H_{\tilde{R}}(q \mid A)=H_{R}\left(-\frac{1}{q} \mid A\right) .
$$

In result for transposition-symmetric diagrams, $\tilde{R}=R$, HOMFLY actually depend on $q$ via the square of the variable $z=\{q\} \equiv q-1 / q$. This has an immediate consequence for its discriminant. By definition, discriminant ${ }_{q^{2}} H$ vanishes whenever the system

$$
\left\{\begin{aligned}
H & =0 \\
\frac{\partial H}{\partial q^{2}} & =0
\end{aligned}\right.
$$

has a solution - and since $q^{2} \frac{\partial H}{\partial q^{2}}=\frac{q^{4}-1}{q^{2}} \cdot \frac{\partial H}{\partial z^{2}}$, we see that $H(q=\sqrt{1} \mid A)=0$ is always a solution. This means that

$$
\text { for } \quad \tilde{R}=R \quad \text { discriminant }_{q^{2}} H_{R} \sim H_{R}(q=1 \mid A) \cdot H_{R}(q=i \mid R) .
$$

This fact can be reinterpreted as follows: if considered as a polynomial with real coefficients, $H_{R}$ with $\tilde{R}=R$ has distinguished set of zeroes, lying at the real axis $z^{2}$. From the point of view of $q^{2}$ this real axis is further separated into three regions: $z^{2}<-2, z^{2}>2$ and 
$-2<z^{2}<2$, where $q^{2}$ lies respectively on negative and positive rays $q^{2}<-1, q^{2}>1$ and unit circle $|q|=1$ (comp. with [45]). Clearly, discriminant discriminant ${ }_{q^{2}}\left(H_{[1]}\right)$ should have zero,corresponding to the bifurcation points $q^{2}= \pm 1$.

Looking at examples, we observe that (5.3) is always true, at least for representations $R=[1],[21],[22]$, where many results are available. Moreover, for the fundamental representation $R=\square=[1]$ the l.h.s. equals the r.h.s. for all knots with the vanishing defect $[31] \delta^{\mathcal{K}}=0$ :

$$
\delta^{\mathcal{K}}=0 \quad \Longrightarrow \quad D_{[1]}^{\mathcal{K}}(A) \equiv \sqrt{\frac{\text { discriminant }_{q^{2}} H_{[1]}^{\mathcal{K}}}{H_{[1]}^{\mathcal{K}}(A \mid q=1) \cdot H_{[1]}^{\mathcal{K}}(A \mid q=i)}}=1 .
$$

We introduced the square root into the definition of $D^{\mathcal{K}}$, because for knots with nonvanishing defect the ratio is a full square(!) — and this seems also true for other symmetric Young diagrams $R$.

Moreover, it factorizes further. In the simplest case of $5_{1}$ with defect one, $D^{5_{1}}=$ $H_{[1]}^{\mathcal{K}}(A \mid q=\xi) \cdot H_{[1]}^{\mathcal{K}}(A \mid q=i \xi)$ with $\xi^{2}=\frac{1 \pm \sqrt{5}}{2} \cdot i$ pure imaginary, i.e. $\xi \sim(1+i)$. (a real number) - however, such simple description of emerging factors fails for more complicated knots.

\section{More on the role of the special polynomials}

One of the two polynomials, which appeared as a factor in discriminant, is the well known special polynomial $H_{R}(q=1 \mid A)$. We remind that the special polynomials at $q^{2}=1$ are always factorized

$$
H_{R}(q=1 \mid A)=H_{[1]}(q=1 \mid A)^{|R|} \quad \forall R .
$$

Likewise Alexander polynomials for the single hook diagrams $R$ satisfy

$$
H_{R}(q \mid A=1)=H_{[1]}\left(q^{|R|} \mid A=1\right) \quad \text { for } \quad R=\left[a, 1^{b}\right] .
$$

The property (6.1) implies that all $H_{R}(q \mid A)$ of a given knot have common zeroes at $q=1$ and at $A$, which are the zeroes of $H_{[1]}(q=1 \mid A)$, i.e. that all the resultants

$$
\text { resultant }_{q^{2}}\left(H_{R}, H_{R^{\prime}}\right) \vdots H_{[1]}(q=1 \mid A) \quad \forall R, R^{\prime} .
$$

In fact the divisibility of resultants and discriminants is richer: for arbitrary knots resultant $\left(H_{k}, H_{l}\right)$ is always divisible by a power of the special polynomial:

$$
\text { resultant }\left(H_{k}, H_{l}\right) \vdots H_{1}(q=1 \mid A)^{\left[\frac{k l+1}{2}\right]} \text {. }
$$


Moreover,

$$
\begin{array}{lll}
r(1,2) & \vdots & H_{1,1} \\
r(1,3) & \vdots & H_{1,1}^{2} H_{1,2} \\
r(1,4) & \vdots & H_{1,1}^{2} \\
r(1,5) & \vdots & H_{1,1}^{3} H_{1,2} \\
r(2,3) & \vdots & H_{1,1}^{3} H_{2,2} \\
r(2,4) & \vdots & H_{1,1}^{4} H_{2,2} \\
r(2,5) & \vdots & H_{1,1}^{5} H_{2,2} \\
r(3,4) & \vdots \quad H_{1,1}^{6} H_{2,2} H_{3,3}^{2} \\
r(3,5) & \vdots \quad H_{1,1}^{8} H_{1,2} H_{2,2} H_{3,3}^{2} \\
r(4,5) & \vdots \quad H_{1,1}^{10} H_{2,2}^{3} H_{3,3}^{2} H_{4,4}^{2}
\end{array}
$$

and

$$
\begin{array}{rll}
d_{1} & \vdots & H_{1,1} H_{1,2} \\
d_{2} & \vdots & - \\
d_{3} & \vdots & H_{1,1}^{2} \\
d_{21} & \vdots & H_{1,1}^{3} H_{21,2} \\
d_{4} & \vdots & H_{1,1}^{4} \\
d_{31} & \vdots & H_{1,1}^{4} \\
d_{22} & \vdots & H_{1,1}^{4} H_{2,2} \\
d_{211} & \vdots & H_{1,1}^{4} \\
d_{1111} & \vdots & H_{1,1}^{4}
\end{array}
$$

where we denoted $H_{Q, j}=H_{Q}\left(q^{2 j}=1\right)$.

From the factorization property we see that

$$
\text { resultant }_{q^{2}}\left(H_{1}, H_{r}\right)=0 \quad \text { if } \quad s p(A)=0
$$

and

$$
\text { resultant }_{q^{2}}\left(H_{2}, H_{r}\right)=0 \quad \text { if } \quad s p(A) \cdot s p\left(A^{2}\right)=0
$$


if and so on. Actually it turns out that these peculiar zeroes of resultants are the limiting points of the sequence of roots. Here is a pictorial and numerical evidence for $7_{1}$-knot: the resultant zeroes in this case

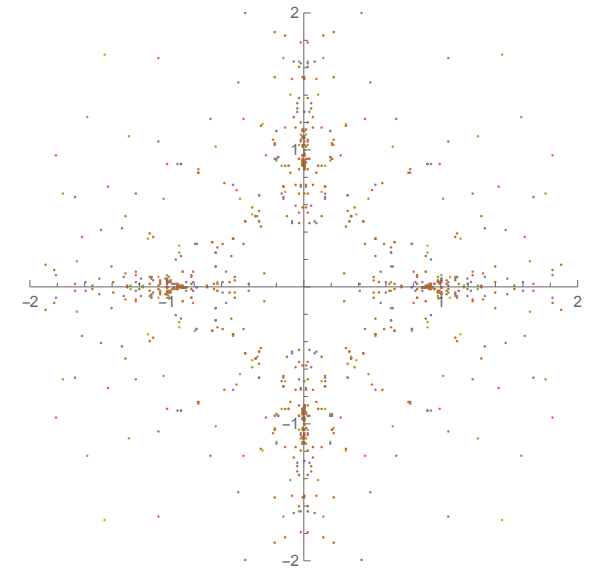

are clearly concentrated near the roots $A= \pm \frac{\sqrt{3}}{2}$ of the special polynomial. For example, resultant $_{q^{2}}\left(H_{1}, H_{7}\right)$ has 414 roots, and 90 of them lie at distances less then 0.2 from the roots of $s p(A)$.

Similar is the distribution of resultant ${ }_{q^{2}}\left(H_{1}, H_{r}\right)$ for the $7_{2}$-knot:
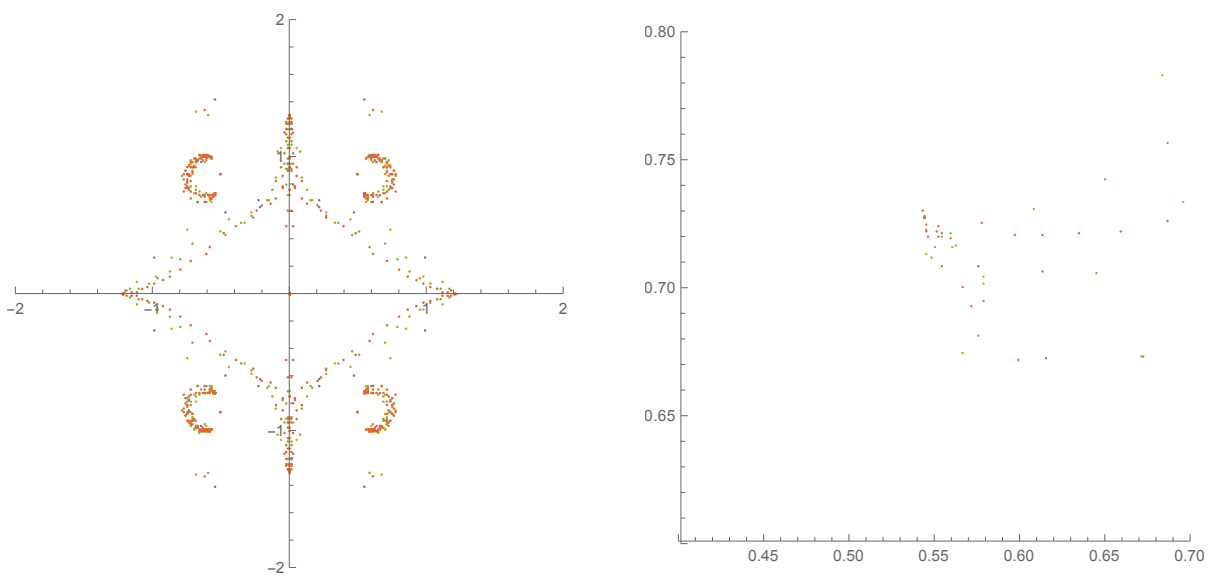

\section{Hopes for divisibility: differential expansion}

As mentioned in the Introduction, the seemingly important property of iterated maps was that $F_{k n}$ always contains $F_{n}$ as a factor - simply because a closed orbit of order $n$ is always an orbit of order $n k$. Though obvious, this fact alone requires a correlation to exist between the roots of $F_{k n}$ and $F_{n}$ - and can therefore play a role in occurrence of the Mandelbrot property. It is a natural question, if similar divisibility can emerge for colored HOMFLY. 
A possible approach to this problem is the study of differential expansion. Namely, for symmetric-representation HOMFLY of any prime knot $\mathcal{K}$ we have [31]:

$$
H_{r}^{\mathcal{K}}=1+[r]\left\{A q^{r}\right\}\{A / q\} \cdot G_{1}^{\mathcal{K}}(q \mid A)+\sum_{j}^{r} \frac{[r] !}{[j] ![r-j] !}\{A / q\} \prod_{i=0}^{j-1}\left\{A q^{r+i}\right\} \cdot G_{j}^{\mathcal{K}}(q \mid A) .
$$

As we just discussed, at $q=1$ all functions $G_{j}$ are such, that (6.1) is true and divisibility takes place. However, for $q \neq 1$ this is no longer so.

Of course, all the differences $H_{r}-1$ have a common zero at $A=q$, but this simply means that of interest can be the ratios

$$
h_{r}=\frac{H_{r}-1}{\{A / q\}} .
$$

Each $h_{r}$ is divisible by $\left\{A q^{r}\right\}$ - and to increase the chances for common zeroes, it deserves switching to either $\tilde{h}_{r}=h_{r}\left(q \mid A q^{-r}\right)$ or to $\hat{h}_{r}=h_{r}\left(q^{r} \mid A\right)$.

We plot zeros for

$$
\operatorname{resultant}_{q^{2}}\left(\frac{H_{i}\left(q^{j}\right)-1}{\left\{A / q^{j}\right\}\left\{A q^{i j}\right\}}, \frac{H_{j}\left(q^{i}\right)-1}{\left\{A / q^{i}\right\}\left\{A q^{i j}\right\}}\right)
$$

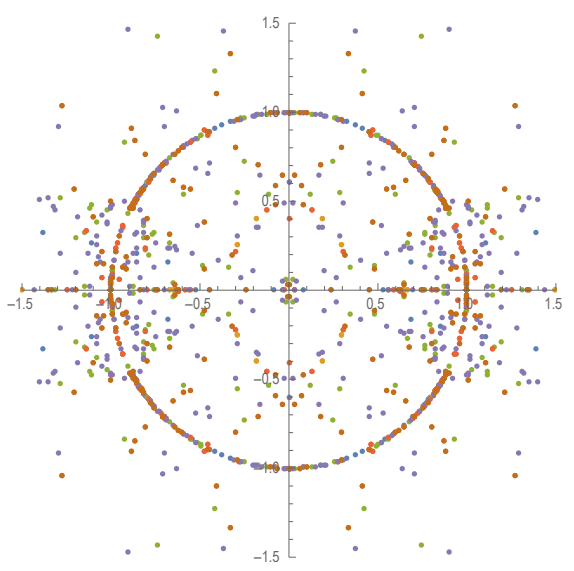

and those for

$$
\operatorname{resultant}_{q^{2}}\left(\frac{H_{i}-1}{\{A / q\}\left\{A q^{i}\right\}}, \frac{H_{j}-1}{\{A / q\}\left\{A q^{j}\right\}}\right)
$$

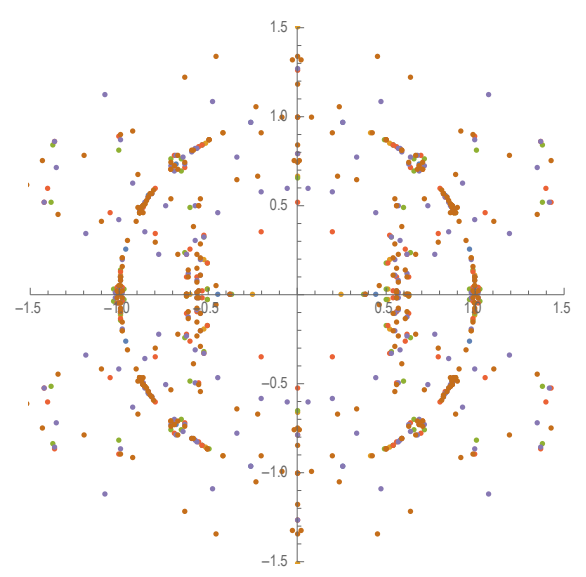




\section{Julia bundle}

Over each point in the $A$-plane we can put a pattern of zeroes of the polynomials $H_{n}$ in $q^{2}$. In the case of Mandelbrot these zeroes of $F_{n}$ (collection of closed orbits) are called Julia sets, and the entire construction is a Julia "bundle" over the Mandelbrot set - we keep the same name in our context. Julia sets are reshuffled at the lines $L_{n}$ and it is instructive to see, how this looks in the case of colored HOMFLY.

We begin with a few illustrative pictures: plot the $q$-roots of $H_{r}^{4_{1}}$ at various values of $A$ for the figure eight knot:

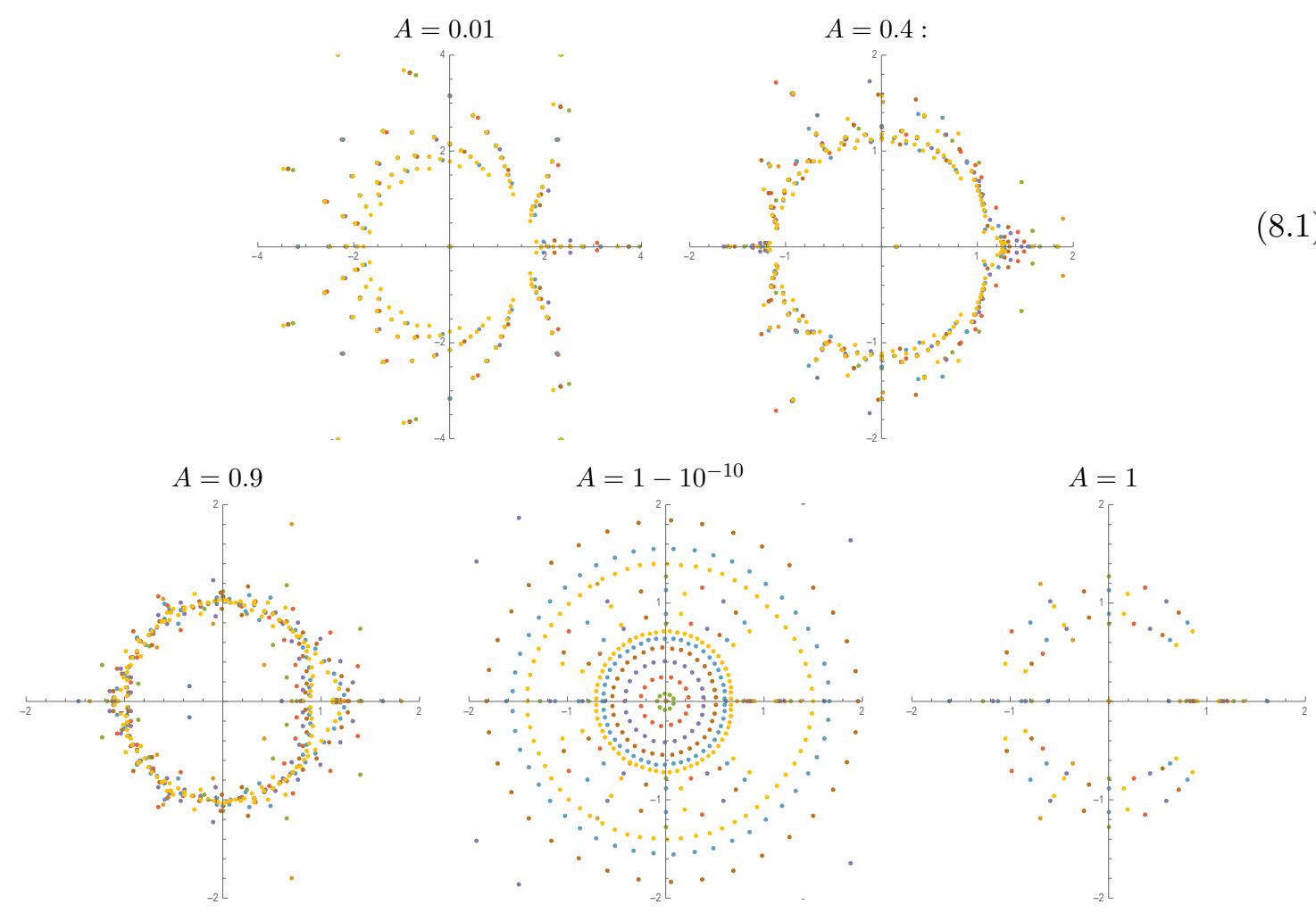

Immediately seen is the special role of the point $A^{2}=1$, where the picture is drastically simplified. This is not a surprise, because at this point the roots of all symmetricallycolored $H_{r}$ are described by (6.2). Namely, they are all expressed through the roots $Q_{\alpha}$ of the Alexander polynomial, whose degree is related to the defect $\delta^{\mathcal{K}}$ of the differential expansion [31]:

$$
\text { roots of } H_{r}(Q \mid A= \pm 1)=\left\{Q_{\alpha}^{1 / r} \cdot e^{2 \pi i j / r}, \alpha=1, \ldots, 2\left(\delta^{\mathcal{K}}+1\right), j=0, \ldots, r\right\} .
$$

For a given $r$ the roots lie on concentric circles, shown in the picture is a collection for various values of $r$ (different $r$ are shown in different colors) - thus the picture looks a little more complicated.

However, a real complication appears when we leave the point $A^{2}=1$. At $A^{2} \neq 1$ the power of $H_{r}$ in $Q$ is usually bigger than $r \times$ that of the Alexander polynomial - and in immediate vicinity of $A= \pm 1$ the extra roots reappear from infinity and zero. Thus the 
$r$-th component of the Julia set in the vicinity of $A= \pm 1$ has a peculiar form of $2 \delta^{\mathcal{K}}+4$ concentric circles round $Q=0$ : two of the $A$-dependent radii $\sim\left(A^{2}-1\right)^{ \pm 1 / \kappa_{r}}$ with $\kappa_{r}$ on each, and $2 \delta^{\mathcal{K}}+2$ circles of radii $Q_{\alpha}^{1 / r}$ with $2\left(\delta^{\mathcal{K}}+1\right)$ points on each. Here

$$
\kappa_{r}=\operatorname{deg}_{Q}\left(H_{r}\right)-r\left(\delta^{\mathcal{K}}+1\right) .
$$

For some knots, including $3_{1}, 5_{1}, 5_{2}, 7_{1}, 7_{2}, 7_{3}, 7_{4}, 7_{5}, \ldots$ all the Alexander roots $Q_{\alpha}$ belong to the unit circle - then all the $A$-independent circles for all $\alpha$ and $r$ merge together and get densely populated.

Despite universality of this pattern, it occurs only in the very close vicinity of $A^{2}=1$ - since $\kappa_{r}$ is typically large, it breaks down at extremely small $A^{2}-1$, like $10^{-r}$ for $4_{1}$. Indeed, the first zero of $\operatorname{discriminant}_{Q}\left(H_{r}^{4_{1}}\right)$, where some roots from the extra two circles collides with those of original ones, occurs already at $A^{2} \approx 0.97$ and $A^{2} \approx 1.03$ for $r=3$.

When $A$ moves away from 1 concentric circles deform and collide with "Alexander" circles (we plot zeros of $H_{10}^{4_{1}}$ ):

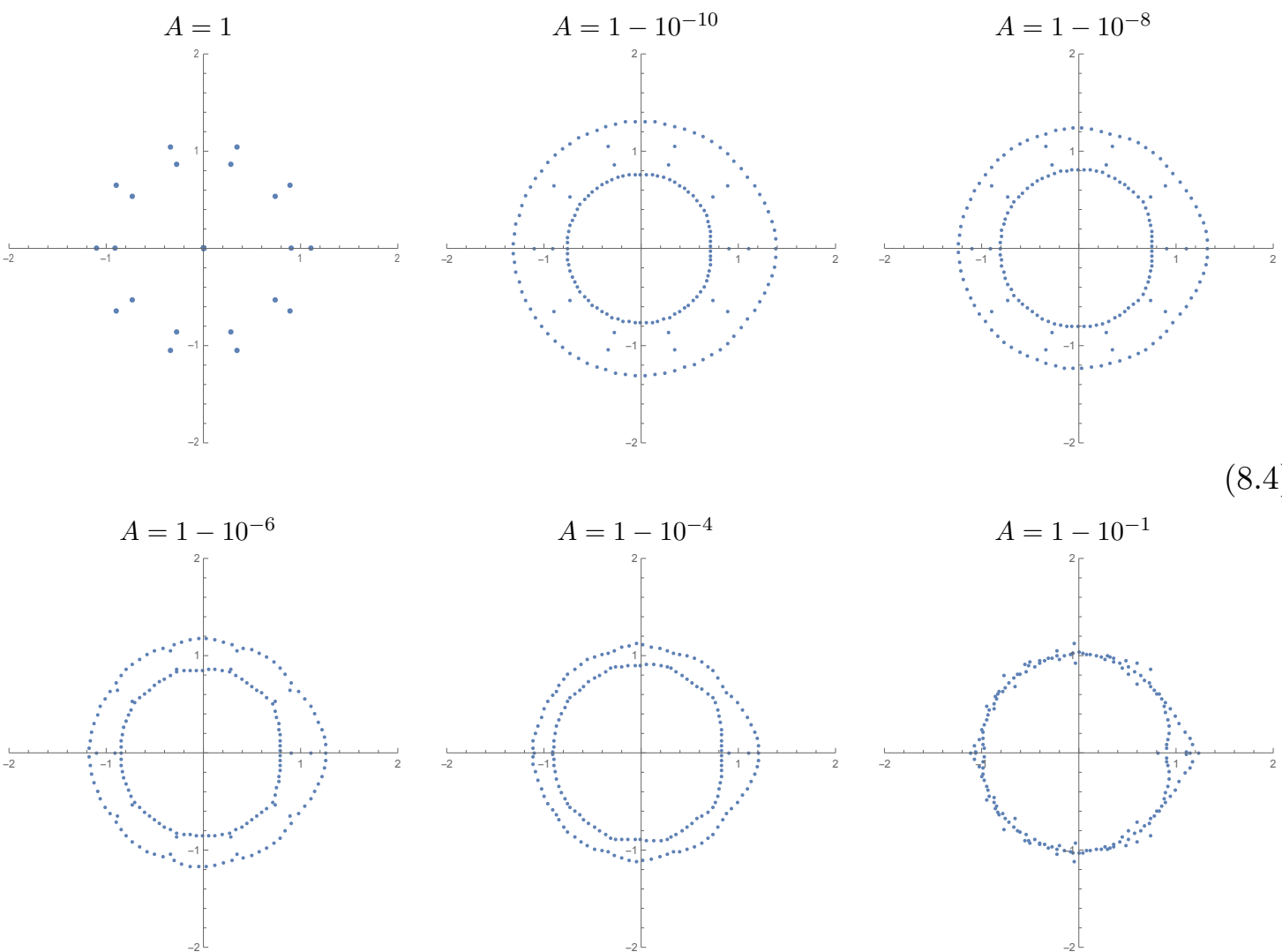


We can also consider perturbation in purely imaginary direction

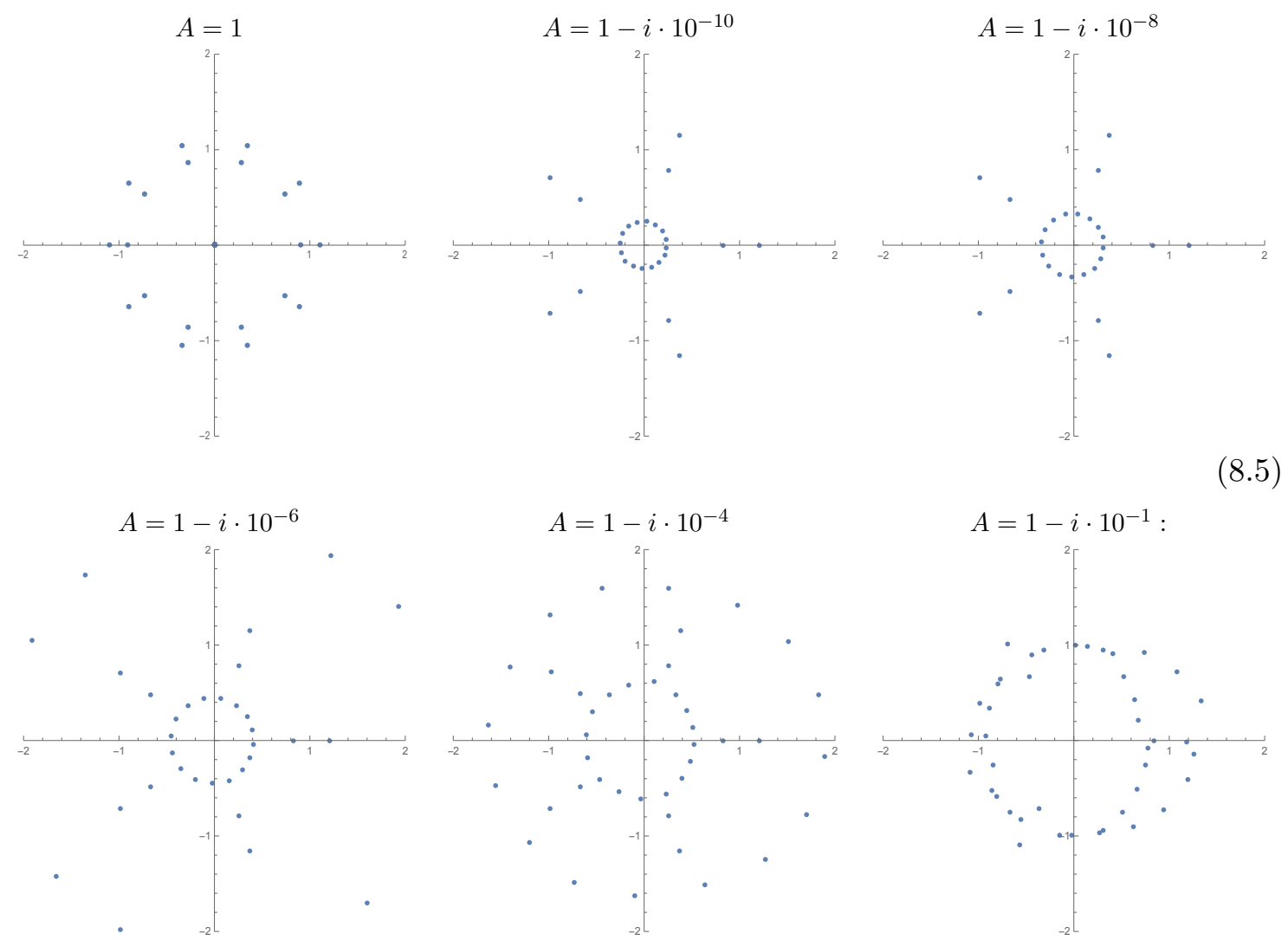

For the trefoil $3_{1}$ all the roots of Alexander polynomial are unimodular and some circles coincide:

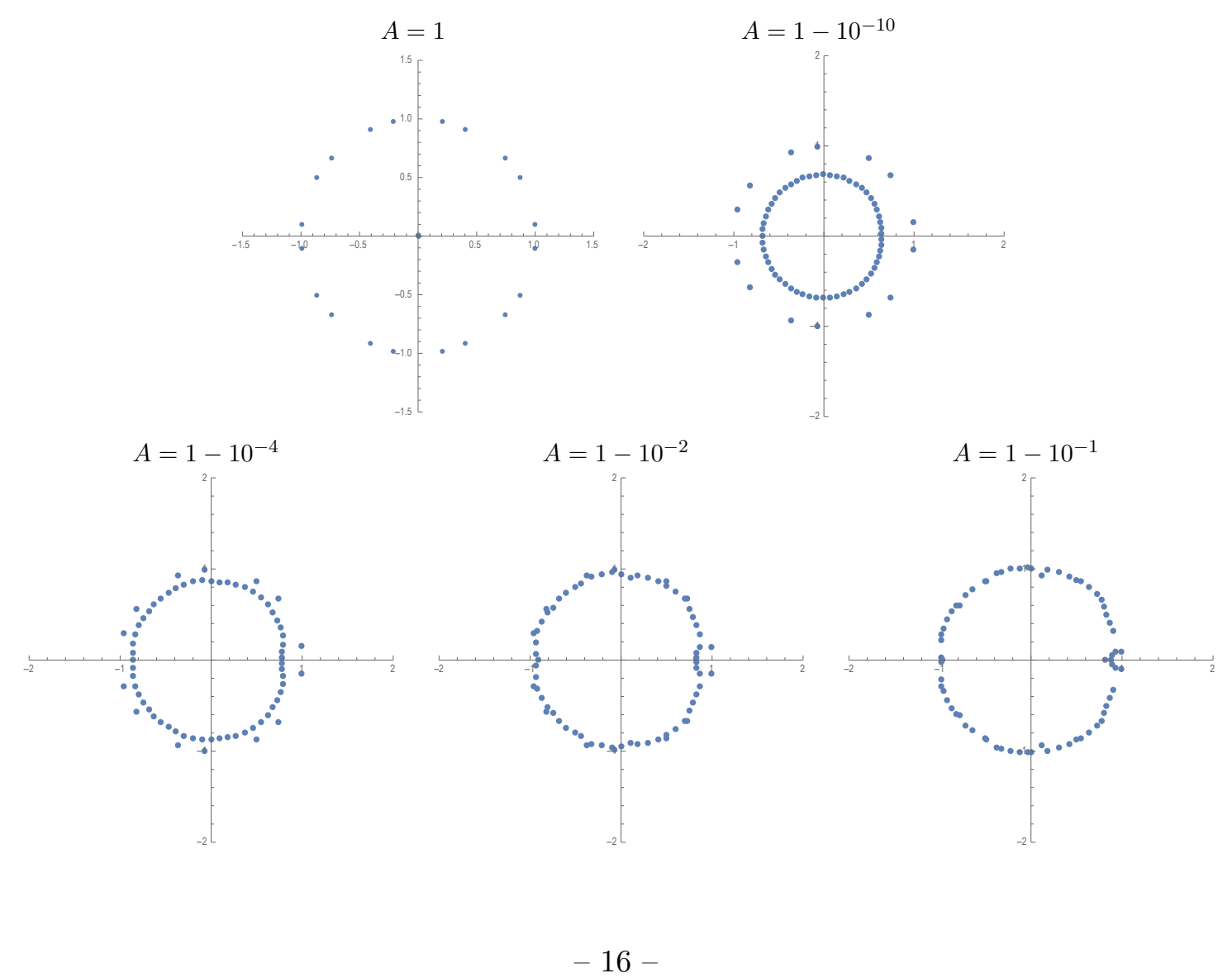


and

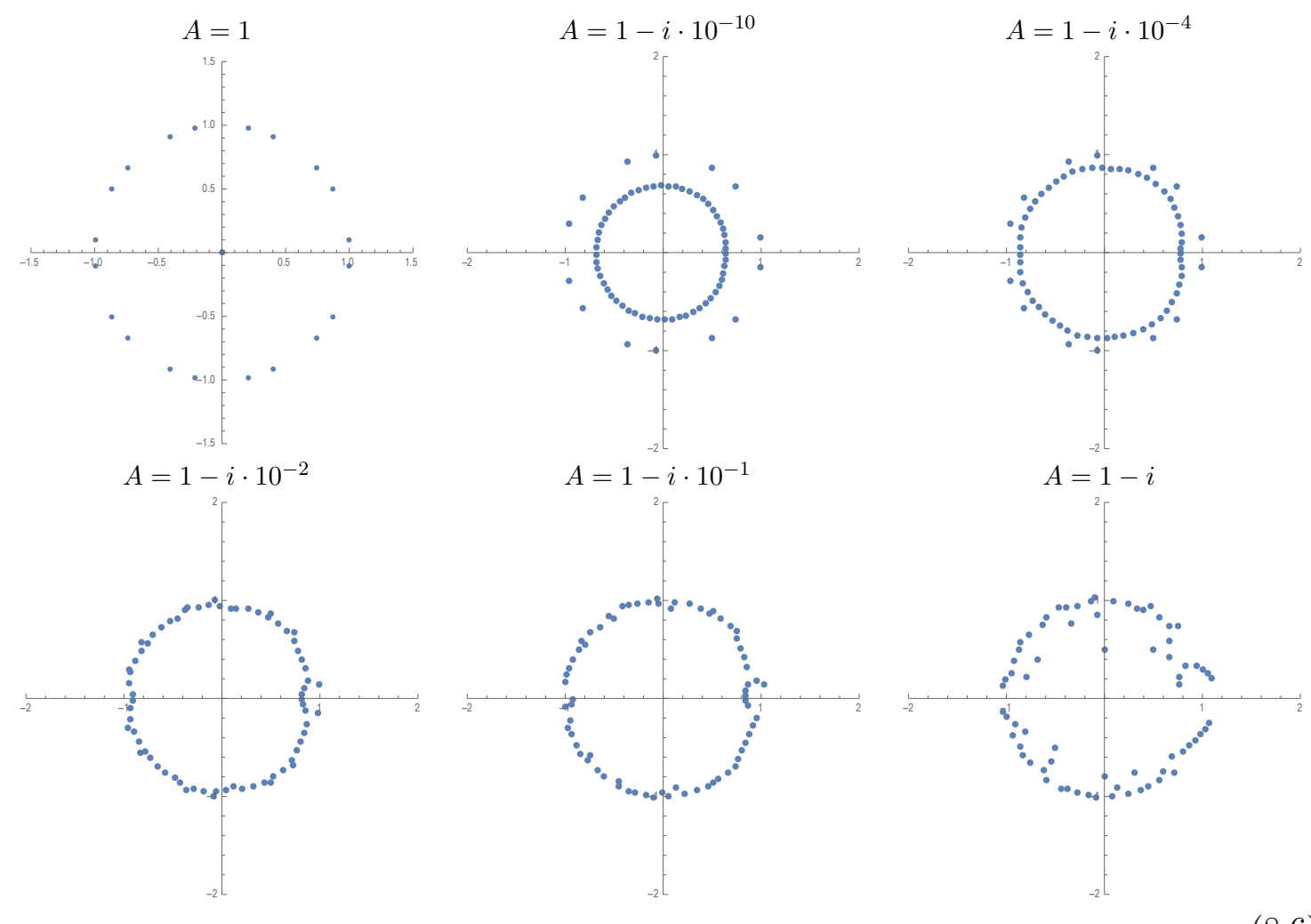

It turns out that if $\left\{q^{r}\right\}=1$ then

$$
\left(\frac{d}{d A}\left(H_{r}\right)\right)_{A=1}=0
$$

This allows us to define

$$
H_{r}=A l\left(q^{r}\right)+\{A\}\left\{q^{r}\right\} P(q)+o(A-1) .
$$

The behavior of concentric circles depends much on the degrees of $A l\left(q^{r}\right)$ and $P(q)$. For $3_{1}$-knot it turns out that each term of expansion with respect to $A-1$ contains the same maximal positive power of $q$, but different negative powers. This is the reason to the fact that there exists a circle collapsing to the origin, but there is not a circle shrinking to infinity. 
We also clearly see a cavity near the point $q=1$. For the $4_{1}$ the picture looks like (we show also the unimodular circle for convenience):

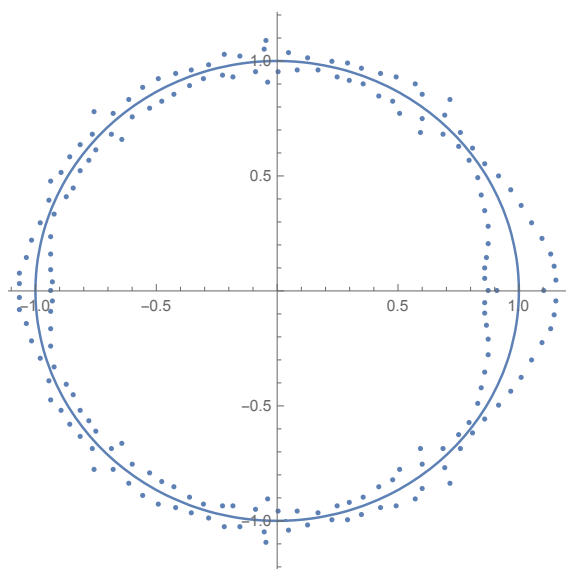

However, when the degrees coincide (say, for $3_{1}$ ), the picture looks differently:

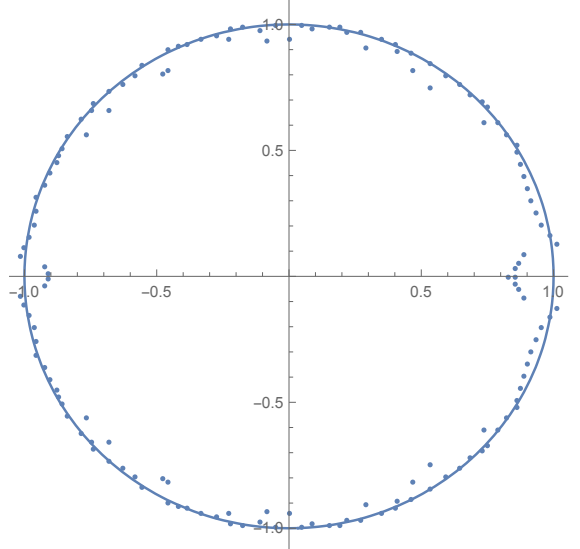

\section{Conclusion}

In this paper we provided some evidence that colored HOMFLY polynomials can exhibit the what we called Mandelbrot property: zeroes of resultant ${ }_{q^{2}}\left(H_{m}, H_{n}\right)$ form structures of codimension one in the complex- $A$ plane. Accuracy of this statement is questionable: emerging curves are at best not as smooth as they were for Mandelbrot sets - nothing to say that we have not any analytical description of those, similar to (1.2). Correlation between zeroes at different $n$ is clearly present, but it still a question, if it is sufficient to exactly provide the Mandelbrot property. Moreover, the quality of "one-dimensionality" seems related to other properties of knots - for example, it looks better for knots with defect zero.

In fact, the choice of HOMFLY $H_{n}$ instead of, say, the differences $H_{n}-1$, for the substitute of the shifted iterated maps, or of $A$ for the role of modulus are rather arbitrary, and these choices affect the pictures - though not as much as one could expect (what is, however, in full accordance with the viewpoint of [11, 12], thus only confirming the possible relation to Mandelbrot theory). 
Further research is needed to understand what really happens. Straightforward computer experiments are not easy - calculation of complicated colored HOMFLY is still a challenge, and finding zeroes of resultants of complicated polynomials is also a difficult task for today's computers. What is needed is better theoretical understanding of HOMFLY polynomials and especially of their factorization properties and zeroes - perhaps, in the spirit of our simple observations in [45].

\section{Acknowledgments}

This work was performed at the Institute for Information Transmission Problems with the financial support of the Russian Science Foundation (Grant No. 14-50-00150).

Open Access. This article is distributed under the terms of the Creative Commons Attribution License (CC-BY 4.0), which permits any use, distribution and reproduction in any medium, provided the original author(s) and source are credited.

\section{References}

[1] B.B. Mandelbrot, Fractal aspects of the iteration of $z \mapsto \lambda z(1-z)$ for complex $\lambda, z$, Ann. New York Acad. Sci. 357 (1980) 249.

[2] B.B. Mandelbrot, The Fractal Geometry of Nature, W. Freeman and Co., New York U.S.A. (1977).

[3] J.W. Alexander, Topological invariants of knots and links, Trans. Am. Math. Soc. 30 (1928) 275 .

[4] J.H. Conway, An Enumeration of Knots and Links, and Some of Their Algebraic Properties, in Computational Problems in Abstract Algebra. Proceedings of a Conference Held at Oxford Under the Auspices of the Science Research Council Atlas Computer Laboratory, 29th August to 2nd September 1967, J. Leech ed., Pergamon Press, Oxford and New York (1970), pp. 329-358.

[5] V.F.R. Jones, Index for subfactors, Invent. Math. 72 (1983) 1 [InSPIRE].

[6] V.F.R. Jones, A polynomial invariant for knots via von Neumann algebras, Bull. Am. Math. Soc. 12 (1985) 103 [INSPIRE].

[7] V.F.R. Jones, Hecke algebra representations of braid groups and link polynomials, Annals Math. 126 (1987) 335 [INSPIRE].

[8] L. Kauffman, State models and the Jones polynomial, Topology 26 (1987) 395.

[9] P. Fréyd, D. Yetter, J. Hoste, W.B.R. Lickorish, K. Millett and A. Ocneanu, A new polynomial invariant of knots and links, Bull. Am. Math. Soc. 12 (1985) 239 [INSPIRE].

[10] J.H. Przytycki and K.P. Traczyk, Invariants of Conway type, Kobe J. Math. 4 (1987) 115.

[11] V. Dolotin and A. Morozov, The Universal Mandelbrot Set. Beginning of the Story, World Scientific (2006).

[12] V. Dolotin and A. Morozov, Algebraic geometry of discrete dynamics. The Case of one variable, hep-th/0501235 [INSPIRE]. 
[13] H. Itoyama, A. Mironov, A. Morozov and A. Morozov, HOMFLY and superpolynomials for figure eight knot in all symmetric and antisymmetric representations, JHEP 07 (2012) 131 [arXiv:1203.5978] [INSPIRE].

[14] H. Itoyama, A. Mironov, A. Morozov and A. Morozov, Character expansion for HOMFLY polynomials. III. All 3-Strand braids in the first symmetric representation, Int. J. Mod. Phys. A 27 (2012) 1250099 [arXiv:1204.4785] [INSPIRE].

[15] http://en.wikipedia.org/wiki/Mandelbrot_set.

[16] A. Morozov, Universal Mandelbrot Set as a Model of Phase Transition Theory, JETP Lett. 86 (2007) 745 [arXiv:0710.2315] [InSPIRE].

[17] V. Dolotin and A. Morozov, On the shapes of elementary domains or why Mandelbrot Set is made from almost ideal circles?, Int. J. Mod. Phys. A 23 (2008) 3613 [hep-th/0701234] [INSPIRE].

[18] V. Dolotin and A. Morozov, Introduction to Non-Linear Algebra, World Scientific (2007) [hep-th/0609022] [INSPIRE].

[19] M.J. Feigenbaum, Quantitative Universality for a Class of Nonlinear Transformations, J. Stat. Phys. 19 (1978) 25 [inSPIRE].

[20] M.J. Feigenbaum, The universal metric properties of nonlinear transformations, J. Stat. Phys. 21 (1979) 669.

[21] L. Landau and E. Lifshitz, Hydrodynamics, section 32, Nauka, Moscow (1986).

[22] A. Anokhina and A. Morozov, Cabling procedure for the colored HOMFLY polynomials, Teor. Mat. Fiz. 178 (2014) 3 [arXiv:1307.2216] [INSPIRE].

[23] P. Dunin-Barkowski, A. Mironov, A. Morozov, A. Sleptsov and A. Smirnov, Superpolynomials for toric knots from evolution induced by cut-and-join operators, JHEP 03 (2013) 021 [arXiv:1106.4305] [INSPIRE].

[24] A. Mironov, A. Morozov and A. Morozov, Character expansion for HOMFLY polynomials. I. Integrability and difference equations, in Strings, Gauge Fields, and the Geometry Behind: The Legacy of Maximilian Kreuzer, A. Rebhan, L. Katzarkov, J. Knapp, R. Rashkov and E. Scheidegger eds., World Scietific Publishins Co. Pte. Ltd. (2013), pp. 101-118 [arXiv: 1112.5754] [INSPIRE].

[25] A. Mironov, A. Morozov and A. Morozov, Character expansion for HOMFLY polynomials. II. Fundamental representation. Up to five strands in braid, JHEP 03 (2012) 034 [arXiv:1112.2654] [INSPIRE].

[26] H. Itoyama, A. Mironov, A. Morozov and A. Morozov, Eigenvalue hypothesis for Racah matrices and HOMFLY polynomials for 3-strand knots in any symmetric and antisymmetric representations, Int. J. Mod. Phys. A 28 (2013) 1340009 [arXiv:1209.6304] [INSPIRE].

[27] H. Fuji, S. Gukov and P. Sułkowski, Super-A-polynomial for knots and BPS states, Nucl. Phys. B 867 (2013) 506 [arXiv:1205.1515] [INSPIRE].

[28] S. Nawata, P. Ramadevi and Zodinmawia, Colored HOMFLY polynomials from Chern-Simons theory, J. Knot Theor. 22 (2013) 1350078 [arXiv:1302.5144] [INSPIRE].

[29] A. Mironov, A. Morozov, A. Morozov, P. Ramadevi and V.K. Singh, Colored HOMFLY polynomials of knots presented as double fat diagrams, JHEP 07 (2015) 109 [arXiv: 1504.00371] [INSPIRE]. 
[30] A. Mironov and A. Morozov, Towards effective topological field theory for knots, Nucl. Phys. B 899 (2015) 395 [arXiv: 1506.00339] [InSPIRE].

[31] Ya. Kononov and A. Morozov, On the defect and stability of differential expansion, JETP Lett. 101 (2015) 831 [arXiv: 1504.07146] [INSPIRE].

[32] N.M. Dunfield, S. Gukov and J. Rasmussen, The Superpolynomial for knot homologies, Exp. Math. 15 (2006) 129 [math.GT/0505662] [INSPIRE].

[33] A. Mironov, A. Morozov and A. Morozov, Evolution method and "differential hierarchy" of colored knot polynomials, AIP Conf. Proc. 1562 (2013) 123 [arXiv:1306.3197] [InSPIRE].

[34] S. Arthamonov, A. Mironov and A. Morozov, Differential hierarchy and additional grading of knot polynomials, Theor. Math. Phys. 179 (2014) 509 [arXiv:1306.5682] [InSPIRE].

[35] Ya. Kononov and A. Morozov, On the defect and stability of differential expansion, JETP Lett. 101 (2015) 831 [arXiv: 1504.07146] [INSPIRE].

[36] I. Gelfand, M. Kapranov and A. Zelevinsky, Discriminants, Resultants and Multidimensional Determinants, Birkhauser (1994).

[37] A. Morozov and S. Shakirov, Analogue of the identity Log Det $=$ Trace Log for resultants, J. Geom. Phys. 61 (2011) 708 [arXiv:0804.4632].

[38] A. Morozov and S. Shakirov, Resultants and contour integrals, Funct. Anal. Appl. 46 (2012) 33 [arXiv:0807.4539].

[39] A. Morozov and S. Shakirov, Introduction to Integral Discriminants, JHEP 12 (2009) 002 [arXiv: 0903.2595] [INSPIRE].

[40] A. Morozov and S. Shakirov, New and Old Results in Resultant Theory, Theor. Math. Phys. 163 (2010) 587 [arXiv:0911.5278] [INSPIRE].

[41] A. Anokhina, A. Morozov and S. Shakirov, Resultant as Determinant of Koszul Complex, Theor. Math. Phys. 160 (2009) 1203 [arXiv:0812.5013] [InSPIRE].

[42] S. Shakirov, Higher discriminants of polynomials, Theor. Math. Phys. 153 (2007) 1477 [math.AG/0609524] [INSPIRE].

[43] KNOT POLYNOMIALS and other link invariants, http://knotebook.org.

[44] Theoretical, Mathematical \&3 String Physics, http://knotebook.org/knotebook/HOMFLY/resultants/resultant.htm.

[45] Ya. Kononov and A. Morozov, Factorization of colored knot polynomials at roots of unity, Phys. Lett. B 747 (2015) 500 [arXiv:1505.06170] [INSPIRE]. 